\title{
1 Physical activity patterns and function three months after arthroscopic partial meniscectomy
}

2 Shane S. Ilich ${ }^{\mathrm{A}^{*}}$, Alasdair R Dempsey ${ }^{\mathrm{B}, \mathrm{C}^{*}}$, Peter M. Mills ${ }^{\mathrm{C}, \mathrm{D}}$, Daina L Sturnieks ${ }^{\mathrm{A}, \mathrm{E}}$, Gwidon W.

3 Stachowiak $^{\mathrm{F}}$, , Ken F. Maguire ${ }^{\mathrm{G},}$ Markus S Kuster $^{\mathrm{H}}$, David G.Lloyd ${ }^{\mathrm{A}, \mathrm{C}, \mathrm{D}}$

$4 \quad *$ Joint First Authors

5 A. School of Sports Science, Exercise and Health, The University of Western Australia, Perth, $6 \quad$ Australia

7 B. School of Chiropractic and Sports Science, Murdoch University, Murdoch, Australia

8 C. Centre for Musculoskeletal Research, Griffith Health Institute, Griffith University, Gold Coast,

$9 \quad$ Australia

10 D. School of Rehabilitation Science, Griffith University, Gold Coast, Australia

11 E. Prince of Wales Medical Research Institute, Randwick, Australia

12 F. School of Mechanical Engineering, The University of Western Australia, Perth, Australia

13 G. Perth Orthopaedic and Sports Medicine Centre, Perth, Australia

14 H. Department of Orthopaedic Surgery, Kantonsspital St. Gallen, St. Gallen 9027, Switzerland

Corresponding Author: $\quad$ Prof David Lloyd

16 Address:

17

18

19

20

21

22

23

24

25

26
Musculoskeletal Research Program, Griffith Health Institute

Clinical Science 1 (G02)

Gold Coast Campus

Griffith University,

Southport, 4222

AUSTRALIA

Telephone:

+61 (7) 55528593

+61 (7) 55528674
david.lloyd@griffith.edu.au
Email:

Word Count: 2979 Number of Tables: 2 Number of Figures: 0 


\section{Abstract}

28 Objectives: To compare physical activity levels, subject-reported function, and knee strength in 21

29 arthroscopic partial meniscectomy (APM) patients (age 45.7 (6.06) years, BMI 27.3(5.96) Female

30 60\%) 3 months post-surgery with 21 healthy controls (age 43.6 (5.71) years, BMI 24.5(4.2) Female

$3160 \%$ ) matched at the cohort level for age, gender and BMI.

32 Design: Case control study

33 Methods: Physical activity intensity, number of steps, and minutes spent in activity wereobjectively

34 quantified using an accelerometer-based activity monitor worn for 7 days. The Knee Injury and

35 Osteoarthritis Outcome Score (KOOS) and concentric quadriceps strength were used to evaluate

36 function post-surgery. Differences in activity levels and functional outcomes between the APM and

37 control participants were assessed using t-tests, while multiple linear regression was used toquantify

38 the best predictors of physical activity.

39 Results: APM patients engaged in a similar duration of activity to controls (469.0 (128.39)minutes

40 vs. 497.1 (109.9) minutes), and take a similar number of steps per day (9227 (2977) vs. 10383 (3501),

41 but performed their activity at lower levels of intensity than controls. Time spent in moderate $\left(r^{2}=\right.$

$420.19)$ and hard $\left(r^{2}=0.145\right)$ intensity physical activity was best predicted by the Symptoms sub-scale of

43 the KOOS for both controls and APM patients.

44 Conclusions: APM patients participate in similar activity however at a lower level, with thereduction

45 in activity at higher intensities related to the presence of symptoms of knee osteoarthritis.

\section{$46 \quad$ Kevwords}

47 Menisectomy; Physical activity; osteoarthritis; function 


\section{$49 \quad$ Introduction}

50

51

52

Arthroscopic partial meniscectomy (APM) is a common knee surgery used to treat meniscal damage of the knee. ${ }^{1-3}$ Despite the surgery being successful in correcting physical dysfunction, ${ }^{4}$ APM can result in limitations in patient-relevant functional outcomes. ${ }^{5} \mathrm{~A}$ common complaint from APM patients is decreased levels of physical activity post-surgery compared to pre-injury. ${ }^{5,6}$ TheKnee Injury and Osteoarthritis Outcome Score (KOOS) is a questionnaire that was specifically designed for younger, more active populations, a similar group to those who commonly undergo APM surgery. ${ }^{7-9}$ Data from KOOS studies has shown that meniscal surgery populations report increased pain and difficulties in participating in sport and recreational activities at 3 months, ${ }^{6} 6-18$ months, ${ }^{10}$ and 4 years ${ }^{5}$ post-operatively. However this questionnaire only assesses difficulty experienced in performing physical activity, and does not quantify how these difficulties affect the intensity andtime spent in these activities.

Research into physical activity levels in knee surgery and knee osteoarthritis populations has typically focused on the number of minutes spent in activity or the number of steps taken. These measures are most commonly recorded from self-reported questionnaires. ${ }^{3,8,11}$ However, physical activity is not only made up of duration and quantity, but involves a third dimension: intensity which is not usually addressed by these questionnaires. Activity monitors can objectively assess activity intensity, along with time spent in activity and number of steps taken. ${ }^{12,13}$

Meniscal surgery has been shown to lead to increased risk of knee osteoarthritis. ${ }^{1,2,14,15}$ APM surgery has also been associated with reduced concentric knee extension strength. ${ }^{16}$ Thisdecreased muscle strength is also associated with the development of knee osteoarthritis. ${ }^{17,} 18$ There is a relationship between decreased muscle strength and decreased levels of physical activity in both the general and knee osteoarthritis populations. ${ }^{5,17}$ This suggests that maintaining healthy physical activity levels may protect against the loss of muscle strength and therefore the development of osteoarthritis. 
KOOS sub-scores with physical activity duration and intensity measured with an accelerometer; ii)

compare daily physical activity duration and intensity between APM and matched control participants; and iii) identify and describe relationships between APM surgery, KOOS, KOOS subscores and physical activity duration and intensity. It was hypothesized that i) activity monitors will be able to objectively quantify the duration, quantity and intensity of physical activity in APM participants; ii) the duration and intensity of APM patients' physical activity will be less thanmatched controls; and iii) those APM patients who report greater levels of pain and difficulty as quantified by the PAIN and SYMPTOMS subscales of the KOOS, will be more likely to show decreased levels of activity.

\section{Methods}

Twenty-one APM patients and 21 controls were manually selected from a large database based on the ability to match two cohorts on sex, BMI and age, although the followingprocedures were undertaken for the entire data set. Matching was performed at this level due to the retrospective creation of the two groups. Primary consideration was given to 1) individuals with complete datasets and 2) gender matching. APM participants had undergone APM for an isolated meniscal tear a mean of 11 (SD 6) weeks prior to data collection and were recruited from a number of metropolitan orthopaedic clinics, while control participants were recruited via community newspaper advertisements. Both APM and CON participants were screened and excluded if they had clinical (surgery reports checked in APM participants) and/or radiographic evidence of knee osteoarthritis, previous or current back, hip, knee, or ankle joint disease, pain, or injury; any form of arthritis; diabetes; cardiac, circulatory, or neurological conditions; multiple sclerosis; stroke; lowerlimb fractures; bone or joint conditions; and any other disease or injury that may affect gait patterns or predispose to knee osteoarthritis. APM participants were also screened according to the following inclusion/exclusion criteria: isolated arthroscopic meniscectomy of one side of the knee only; no damage to anterior cruciate, medial or lateral collateral ligaments; maximum of one chondraldefect $<2 \mathrm{~cm}$ on the tibial and fibular surfaces, as assessed by the surgeon during arthroscopy; no previous 
101

102

103

104

105

106

107

108

109

110

111

112

113

114

115

116

117

118

119

120

121

122

123

124

125

126

medically documented injuries or surgeries to the knee ligament, cartilage or meniscus; and aged between 35-55 years and BMI $<30$. This study was approved by the University of Western Australia Human Research Ethics Committee, and all participants provided informed, written consent.

Daily physical activity levels were recorded using an Actigraph AM7164-2.2 (Actigraph, Pensacola, FL, USA) physical activity monitor. The Actigraph contains a uniaxial accelerometer which detects vertical accelerations between 0.05 and 2 G. Sampling epoch was set at 60 seconds for this study. The validity and reliability of the Actigraph physical activity monitor has previouslybeen demonstrated. ${ }^{13,19,20}$ Each participant wore the Actigraph on an adjustable belt that was secured firmly around the waist for seven consecutive days. Waist placement was chosen for two reasons. Ithas been validated ${ }^{1321}$ and it enables direct comparison with previous studies that have investigated physical activity in early knee $\mathrm{OA}^{22,23,24 .}$

For each participant, the mean daily duration of activity in minutes, and the mean number of minutes per day spent in light, moderate, and hard activity levels were calculated. Activity levels were defined by accelerometer counts, downloaded using Actilife $\mathrm{X}$ and parameterised using custom Matlab (Mathworks, Natick, MA, USA) scripts in which hard activities were defined by greaterthan 5725 counts/min (6.0 METS), moderate activities were between 1953 and 5724 counts/min (3.0 5.99 METS), while light activities were between 5 and 1952 counts/min (<2.99METS). ${ }^{25}$ Mean daily step count information from the accelerometer was also analysed. Activity data from individualdays were visually inspected to identify days in which the accelerometer was not worn. Allincluded participants had 7 valid days of accelerometer data.

Knee pain and function was scored using the KOOS questionnaire, previously determined as being appropriate to assess a younger and more active population. ${ }^{7-9}$ The KOOS is aself-administered questionnaire that groups items into the following subscales: PAIN; SYMPTOMS; Activities of Daily Living (ADL); Sport and Recreation (S\&R); and Quality of Life (QOL). Each item of the KOOShas a five point Likert-type scale from 0 to 4 . Knee pain and function scores were created from the responses for items in the respective KOOS subscales. These were summed to give a subscalescore, 
and transformed to a normalised 0 to 100 scale, with a score of 100 indicating normal function and a score of zero indicating difficulties. Normalised scores for each of the 5 subscales were used in the subsequent analyses, as well as the overall KOOS score, which was the average of all subscale scores as per previously published use of the KOOS questionnaire. ${ }^{9}$

Height and body mass were measured and BMI calculated from these values. In addition, the participants' maximum isometric and isokinetic knee extension (quadriceps) strength was measured at $180^{\circ}$ s across the range of $0^{\circ}$ to $90^{\circ}$ of knee flexion using a Biodex isokinetic dynamometer (Chattanooga, Shirley, NY, USA). Participants repeated each strength test three times, with thebest effort used for analysis. Peak concentric quadriceps strength was normalised by dividing bybody mass $\times$ height (kg.m).

Meteorological data were acquired for each date an activity monitor was worn by a participant, and included as covariates to eliminate any confounding effects of weather on activity levels. ${ }^{26}$ Specifically, maximum temperature (MAX; degrees Celsius) and rainfall (RAIN; mm)were selected as the two climate variables with the greatest potential to affect physical activity levels.

\section{$\underline{\text { Statistical Analysis }}$}

Statistical data analyses were performed using SPSS version 16.0 for Windows (SPSS Inc., Chicago). Physical activity duration, KOOS, and KOOS sub-scores were compared between the CON and APM groups using independent samples t-tests. Prior to undertaking statistical testing the data was assessed for normality. The associations between KOOS subscales and physical activity intensity level were assessed using pearson product-moment correlations, to investigate relationships between subjective self-report of difficulty performing activity matched objective measures of intensityand time. Finally a backwards stepwise linear regression was performed on the APM participants to identify the most important variable affecting those physical activity levels found to besignificantly different from the control group, with the following variables entered as predictors: age; BMI; sex; maximum daily temperature; rainfall; quadriceps concentric strength; and KOOS sub-scales SYMPTOMS and PAIN. Significance was set at $p<0.05$ for all analyses. 


\section{Results}

154

155

156

157

158

159

160

161

162

163

164

165

166

167

168

169

170

171

172

173

174

175

176

177

No statistical differences in age, BMI, quadriceps concentric strength, minutes spent in light activity, or mean number of steps per day were found between APM patients and controls (Table1). Independent samples t-tests identified significant differences for number of minutes spent in moderate and hard physical activity, as well as for the overall KOOS score and each of its subscales (Table 1), indicating the two groups were differentiated only by the intensity of physical activity and knee function.

Light physical activity was not significantly correlated with any of the KOOS scales.

Moderate physical activity was positively correlated with Symptoms, S \& R, QOL and overall KOOS score (Table 2). Hard physical activity was shown to correlate with Pain, Symptoms, QOL and overall KOOS score. SYMPTOMS emerged as the only significant predictor variable for both the number of minutes spent in moderate activity, $(\mathrm{R}$-squared $=0.149, \mathrm{p}=0.015)$ and the number of minutes spent in hard activity (R-squared $=0.145, \mathrm{p}=0.017$ ).

\section{Discussion}

The first general aim of this study was to examine relationships between KOOS and KOOS sub-scores and physical activity duration and intensity in otherwise healthy persons who had undergone APM for an isolated meniscal tear. Physical activity monitors have been shown to have greater reliability and accuracy in recording physical activity than surveys. ${ }^{27,28}$ The current results showed that no KOOS score was significantly correlated to every day, light intensity activity. Significant correlations were only shown at higher levels of intensity for those sub-scales of the KOOS most likely to be associated with more vigorous activity or pain and discomfort. The poor correlation between the KOOS and activity monitors, particularly for ADL and S\&R subscales, suggest they are not directly quantifying the same factor. The efficacy of the KOOS in accurately identifying changes in, and factors affecting, actual levels of physical activity in APM patientsis therefore questionable. 
It is not possible to derive specific information regarding the duration, quantity or intensity of physical activity by APM patients from the KOOS questionnaire. This data however is providedby the activity monitor. Whilst it was able to differentiate between APM patients and controls inregards to the amount of difficulty involved in performing activities, due to the KOOS design it couldnot identify how the activity levels of those APM patients were different to the controls. Future investigations into the exercise and activity levels of APM patients will need to take this into account. This can be achieved by using accelerometry to directly measure physical activity, and the KOOS questionnaire as a more general overview of broad function and symptoms. APM patients and controls, and to identify those factors influencing activity levels. It was found that for the mean number of STEPS per day, minutes spent in LIGHT activity, and total TIME spent in activity, there were no significant differences between the two groups. This indicates that APM patients engage in similar quantity (steps) and duration (total time) of basic physical activity, and perform similar levels of daily activities at light intensity. What did differentiate the APM fromthe control participants were the minutes spent in MODERATE and HARD activity, with the APM patients found to spend significantly less time engaged in each level of intensity. Thus, it would appear that APM patients, while engaging in similar exercise/daily activity routines to non-surgery controls, do not perform that activity to the same level of intensity, remaining instead at the lower, light level of intensity. Significant differences were also found for each of the KOOS measure subscales, particularly S\&R and QOL, indicating that it was higher-intensity activities such as sport that caused APM patients more difficulty. This results are similar to those found by Thorlund and colleagues $^{29}$ in a APM population at 2 years. A possible confounder is that the ADL subscale of the

200 KOOS also yielded a statistical difference between the two populations. This may mean that whilst 201 the APM patients reported more discomfort engaging in daily activities through the KOOS theystill 202 performed them. This is reflected in similar results at light intensities recorded by the activitymonitor. 
hold true across the entire sample population, with those with increased symptoms of knee dysfunction being less likely to engage in higher intensity activities. This would havepossible rehabilitation and treatment ramifications, as programs may need to be tailored to take into account the relative intensity of a recovery exercise, and how this will affect adherence by the patient.

Whilst there was not a significant difference in strength between the APM patients and controls, APM patents have been shown in the literature to be weaker than healthyindividuals. ${ }^{16,29,30}$

211 This includes work published from the lager cohort from which the current study's population was drawn. ${ }^{16}$ Given the relationship between physical activity levels and muscle strength in knee osteoarthritis patients, ${ }^{31,32}$ the link between APM surgery and knee osteoarthritis development, ${ }^{33}$ and

214 the recent suggestion that knee extension strength may play a role in facilitating the development of knee osteoarthritis following APM surgery, ${ }^{16}$ these results may offer an insight as to how this muscle weakness could develop within APM patients. Individuals who undergo APM surgery may not participate in physical activity at sufficient intensity to maintain or improve muscle strengthpostsurgery. Individuals who have underdergone partial meniscectomy tend have maintained quadriceps weakness at six months following surgery, ${ }^{34}$ with strength decrements reported up to four years post surgery. ${ }^{5}$ However the nature of this study makes it unable to provide conclusive evidence on this hypothesis. As only one time point was measured it may be possible that strength had, 1) recovered to normal levels following 3 months, or 2) may subsequently decline, particularly in thosepatients who go onto develop knee joint osteoarthritis. Further work is needed to provide stronger evidence for a relationship between physical activity and quadriceps strength. This should included both a larger sample size and ideally be of longitudinal design.

To date this is the only study that we are aware has used an objective measure of actual physical activity, particularly intensity, on an APM population, in conjunction with asurrogate measure such as the KOOS. These results not only offer support for the use of objective measures of activity such as accelerometers with APM patients, but also provide information regarding the specific activity patterns of this population. Non-participation in higher intensity activity suchas sport, whilst most likely being due to patients consciously or subconsciously protecting the affected 
232 joint, ${ }^{35,}{ }^{36}$ could also have detrimental repercussions on the strength and functional rehabilitation of the

233 joint following APM. ${ }^{5}$ Similarly, participants who reported increased symptoms of knee pain and

234 dysfunction were less likely to participate in higher intensity activity, regardless of whether theywere

235

236

237

238 an APM patient or control participant. Future investigations into the rehabilitation of APMpatients will need to take into account this reduced activity intensity, and the associated potential for a loss of muscle strength around the knee. This could be achieved by consistently implementing a strengthbuilding intervention post-surgery. This work will need to be accompanied by work investigatingthe role that increased exercise intensity plays on patient symptoms and recovery time. Other factors that may have a potential influence on actual physical activity and overall function, including physiological factors such a fear or re-injury or low expectations based on clinician information.

This study was cross-sectional investigation of arthroscopic partial meniscectomy patients $<12$ weeks post-surgery, making it unable to define direct, causative relationships between factors affecting activity levels. Included patients were aged 35-55, meaning the results of this study are valid for a younger, active pre-osteoarthritic sample. We included patients with either medial orlateral meniscectomies in the analysis, which is generally consistent with previous methods and allows these results to be compared to existing literature. ${ }^{1,2,10}$ Cohorts were also not matched on occupation. As occupation has the potential to influence activity and function, this factor should be included infuture studies. A final limitation of the study is the small sample size utilised. This has the potential tolimit the predictive ability of the regression, however we believe that the results from theregression provide important information regarding potential reasons for reduced activity in APM populations. This information can be used to drive both future research and clinicians.

\section{Conclusions}

Persons who had undergone APM 8 to 12 weeks performed a similar amount of physical activity as controls when matched for age, BMI and sex at the cohort level, however spent less time at moderate and high physical activity levels. Time spent by APM participants in moderate and hardintensity levels of activity was best predicted by the SYMPTOMS subscale of the KOOS. 
258

259

260

261

262

263

264

265

266

267

268

269

270

271

272

273 Dr Keith Holt, Dr Greg Witherow, Dr Greg Janes, Dr Peter Annear, Dr Hari Goonatillake, DrDermot

274 Collopy, Dr David Colvin, Dr Peter Campbell.

- Accelerometry provides more detail on physical activity in patients who have undergone APM than activity data from KOOS, in particular exercise intensity. However Pain and Symptoms subscales on KOOS provide important information as to reasons behind changes in physical activity.

- Those who have undergone AMP have the same number of total daily steps as healthy controls but have reduced activity at higher intensity levels. Practitioners should take this into account when designing rehabilitation programs.

- Time spent in higher levels of activity is best predicted by subjectively reported symptoms. Reducing or treating knee symptoms in patients who have undergone APM may allow them to undertake higher intensity physical activity.

\section{Acknowledgments}

We acknowledge the financial support of the NHMRC and Western Australian Medical Research Infrastructure Fund. Thanks to Ms Catherine Hill and Ms Carlee Ackland for assistance with data collection and processing. Thanks to the following surgeons for their support in patient recruitment: 


\section{$\underline{\text { References }}$}

277

278

279

280

281

282

283

284

285

286

287

288

289

290

291

292

293

294

295

296

297

298

299

300

301

302

1. Cicuttini FM, Forbes A, Wang Y, et al. Rate of Knee Cartilage Loss After Partial Meniscectomy. J Rheumatol. 2002;29:1954-6.

2. Williams R, Warner K, Petrigliano F, et al. MRI Evaluation of Isolated Arthroscopic Partial Meniscectomy Patients at a Minimum Five-Year Follow-up. HSS Journal. 2007;3(1):35-43.

3. Meredith DS, Losina E, Mahomed NN, et al. Factors predicting functional and radiographic outcomes after arthroscopic partial meniscectomy: A review of the literature. Arthroscopy. 2005;21(2):211-23.

4. Johnson MJ, Lucas GL, Dusek JK, et al. Isolated Arthroscopic Meniscal Repair: A Long-Term Outcome Study (More Than 10 Years). Am J Sport Med. 1999;27(1):44-9.

5. Ericsson YB, Roos EM, Dahlberg L. Muscle strength, functional performance, and self-reported outcomes four years after arthroscopic partial meniscectomy in middle-aged patients. Arthritis Care Res. 2006;55(6):946-52.

6. Roos EM, Roos HP, Ryd L, et al. Substantial disability 3 months after arthroscopic partial meniscectomy: a prospective study of patient-relevant outcomes. Arthroscopy. 2000;16(6):61926.

7. Roos EM, Toksvig-Larsen S. Knee injury and Osteoarthritis Outcome Score (KOOS) - validation and comparison to the WOMAC in total knee replacement. Health Qual Life Outcomes.

$$
\text { 2003;1(1):17. }
$$

8. Roos EM, Roos HP, Lohmander LS, et al. Knee injury and Osteoarthritis Outcome Score (KOOS) - development of a self-administered outcome measure. J Orthop Sports Phys Ther. 1998;28(2):88-96.

9. Roos EM, Roos HP, Ekdahl C, et al. Knee Injury and Osteoarthritis Outcome Score (KOOS) validation of a Swedish version. Scand J Med Sci Sports. 1998;8(6):439-48.

10. Katz JN, Meredith DS, Lang P, et al. Associations among preoperative MRI features and functional status following arthroscopic partial meniscectomy. Osteoarthritis Cartilage. 2006;14(5):418-22. 
11. Friedenreich CM, Courneya KS, Neilson HK, et al. Reliability and Validity of the Past Year Total Physical Activity Questionnaire. Am J Epidemiol.2006;163(10):959-70.

12. Patterson SM, Krantz DS, Montgomery LC, et al. Automated physical activity monitoring: validation and comparison with physiological and self-report measures. Psychophysiology. 1993;30(3):296-305.

13. King GA, Torres N, Potter C, et al. Comparison of activity monitors to estimate energy cost of treadmill exercise. Med Sci Sports Exerc. 2004;36(7):1244-51.

14. Mills PM, Wang Y, Cicuttini FM, et al. Tibio-femoral cartilage defects 3-5 yearsfollowing arthroscopic partial medial meniscectomy. Osteoarthritis Cartilage. 2008;16(12):1526-31.

15. Wang Y, Dempsey AR, Lloyd DG, et al. Patellofemoral and tibiofemoral articular cartilage and subchondral bone health following arthroscopic partial medial meniscectomy. Knee Surg Sports Traumatol Arthrosc. 2012;20(5):970-8.

16. Sturnieks DL, Besier TF, Hamer PW, et al. Knee strength and knee adduction moments following arthroscopic partial meniscectomy. Med Sci Sports Exerc. 2008;40(6):991-7.

17. Mikesky AE, Mazzuca SA, Brandt KD, et al. Effects of strength training on the incidenceand progression of knee osteoarthritis. Arthritis Care Res. 2006;55(5):690-9.

18. Slemenda C, Brandt KD, Heilman DK, et al. Quadriceps Weakness and Osteoarthritis of the Knee. Ann Intern Med. 1997;127(2):97-104.

19. Freedson PS, Melanson E, Sirard J. Calibration of the Computer Science and Applications, Inc. accelerometer. Med Sci Sports Exerc. 1998;30(5):777-81.

20. Welk GJ, Blair SN, Wood K, et al. A comparative evaluation of threeaccelerometry-based physical activity monitors. Med Sci Sports Exerc. 2000;32(9 Suppl):S489-97.

21. Colbert LH, Matthews CE, Havighurst TC, et al. Comparative Validity of Physical Activity Measures in Older Adults. Med Sci Sports Exerc. 2011;43(5):867-76.

22. Farr JN, Going SB, Lohman TG, et al. Physical activity levels in patients with early knee osteoarthritis measured by accelerometry. Arthritis Rheum. 2008;59(9):1229-36. 
23. Farr JN, Going SB, McKnight PE, et al. Progressive resistance training improves overall physical activity levels in patients with early osteoarthritis of the knee: a randomized controlled trial. Phys Ther. 2010;90(3):356-66.

24. Song J, Semanik P, Sharma L, et al. Assessing physical activity in persons with knee osteoarthritis using accelerometers: data from the osteoarthritis initiative. Arthritis Care Res. 2010;62(12):1724-32.

25. Freedson PS, Melanson E, Sirard J. Calibration of the Computer Science and Applications, Inc. accelerometer. Med Sci Sports Exerc. 1998;30(5):777-81.

26. Brandon CA, Gill DP, Speechley M, et al. Physical activity levels of older community-dwelling adults are influenced by summer weather variables. Applied Physiology Nutrition and Metabolism. 2009;34(2):182-90.

27. Timperio A, Salmon J, Crawford D. Validity and reliability of a physical activity recall instrument among overweight and non-overweight men and women. J Sci Med Sport. 2003;6(4):477-91.

28. Chau JY, Van der Ploeg HP, Dunn S, et al. Validity of the Occupational Sitting and Physical Activity Questionnaire. Med Sci Sports Exerc. 2012;44(1):118-25.

29. Thorlund JB, Aagaard P, Roos EM. Thigh muscle strength, functional capacity, andself-reported function in patients at high risk of knee osteoarthritis compared with controls. Arthritis Care Res. 2010;62(9):1244-51.

30. Durand A, Richards CL, Malouin F. Strength recovery and muscle activation of theknee extensor and flexor muscles after arthroscopic meniscectomy. A pilot study. Clin Orthop. 1991(262):210-26.

31. Heiden TL, Lloyd DG, Ackland TR. Knee extension and flexion weakness in people with knee osteoarthritis: is antagonist cocontraction a factor? J Orthop Sports Phys Ther. 2009;39(11):80715.

32. Hurley MV. The role of muscle weakness in the pathogenesis of osteoarthritis. Rheum Dis Clin North Am. 1999;25(2):283-98, vi. 
33. Bolano LE, Grana WA. Isolated arthroscopic partial meniscectomy. Functional radiographic evaluation at five years. Am J Sport Med.1993;21(3):432-7.

358 34. McLeod M, Gribble P, Pfile K, et al. Effects of arthroscopic partial meniscectomy onqadricaps strength: a systematic review. J Sport Rehabil. Pub Ahead of Print.

35. Stevens J, Mizner R, Snyder-Mackler L. Quadriceps strength and volitional activation beforeand after total knee arthroplasty for osteoarthritis. J Orthop Res. 2003;21(5):775-9.

36. Hede A, Hempel-Poulsen S, Jensen JS. Symptoms and level of sports activity of patients awaiting arthroscopy for meniscal lesions of the knee. J Bone Joint Surg Am.1990;72(4):550-2. 
365 Table 1. Descriptive statistics and t-test results control group and arthroscopic partialmeniscectomy

366 group.

\begin{tabular}{lcc|cc|c} 
& \multicolumn{2}{l|}{ CON subset } & \multicolumn{2}{l}{ APM subset } & \\
\cline { 2 - 5 } & Mean & SD & Mean & SD & p \\
\hline Age (yrs) & 43.6 & 5.7 & 45.7 & 6.1 & 0.299 \\
Sex (\% of females) & 60 & - & 60 & - & \\
BMI (kg/m²) & 24.5 & 4.2 & 27.3 & 6.0 & 0.137 \\
QOL & 95.3 & 8.6 & 53.6 & 17.6 & $<0.001$ \\
S \& R & 98.5 & 4.0 & 53.6 & 25.9 & $<0.001$ \\
ADL & 99.4 & 1.6 & 87.3 & 15.4 & 0.001 \\
Symptoms & 94.5 & 8.9 & 76.2 & 10.2 & $<0.001$ \\
Pain & 98.1 & 4.1 & 82.0 & 8.4 & $<0.001$ \\
KOOS & 97.2 & 4.6 & 70.5 & 12.3 & $<0.001$ \\
Light Activity (mins/day) & 423.6 & 118.2 & 471.8 & 104.3 & 0.196 \\
Moderate Activity (mins/day) & 39.6 & 16.2 & 24.1 & 15.5 & 0.003 \\
Hard Activity (mins/day) & 6.3 & 10.6 & 1.2 & 2.5 & 0.039 \\
Total Activity (mins/day) & 497.1 & 109.9 & 469.0 & 128.4 & 0.542 \\
Steps per day & 10383 & 3501 & 9227 & 2978 & 0.347 \\
Peak Concentric Quadriceps & 0.60 & 0.14 & 0.48 & 0.24 & 0.329 \\
Strength (N/kg*m) & & & & & \\
\hline
\end{tabular}

367 BMI - Body Mass Index; KOOS- Knee Osteoarthritis outcome Scale; The following are KOOS

368 subscales: QOL - Quality of Life; S\&R - Sport and Recreation; ADL - Activities of Daily Living. 
370 Table 2. Significant Pearson correlations between actigraph physical activity levels and KOOS

371 questionnaire sub-scales for both APM patients and control participants.

\begin{tabular}{lccc}
\cline { 2 - 4 } & Light activity & Moderate activity & Hard activity \\
\hline Pain & -0.173 & 0.262 & $0.326^{*}$ \\
Symptoms & -0.064 & $0.381^{*}$ & $0.366^{*}$ \\
ADL & -0.131 & 0.293 & 0.188 \\
S \& R & -0.021 & $0.424^{* *}$ & 0.287 \\
QOL & -0.041 & $0.456^{* *}$ & $0.331^{*}$ \\
KOOS & -0.079 & $0.433^{* *}$ & $0.338^{*}$ \\
\hline
\end{tabular}

* denotes $p<0.05$

** denotes $p<0.01$

372 KOOS- Knee Osteoarthritis outcome Scale; The following are KOOS subscales: QOL - Quality of

373 Life; S\&R - Sport and Recreation; ADL - Activities of Daily Living. 